\title{
Efectos de la roturación del suelo y fertilización con fósforo en el crecimiento inicial de plantaciones de Pinus taeda, en suelos rojos del Noreste de la provincia de Corrientes, Argentina*
}

\author{
Soil ripping and phosphorus fertilization effects on Pinus taeda initial growth in red \\ soils of Northeast Corrientes Province, Argentina \\ CESAR IBAÑEZ1 ${ }^{1}$ PATRICIO NUÑEZ², RAUL PEZZUTTI ${ }^{3}$, FRANCISCO RODRIGUEZ ${ }^{4}$ \\ ${ }^{1}$ Ingeniero Forestal, Temuco, Chile. E-mail: ciban002@yahoo.com \\ ${ }^{2}$ Departamento de Ciencias Forestales, Universidad de La Frontera, Temuco, Chile. E-mail: pnunez@ufro.cl \\ ${ }^{3}$ Forestal Bosques del Plata S.A. Posadas, Argentina. E-mail: rpezzutti@cmpc.com.ar \\ ${ }^{4}$ Forestal Mininco S.A., Los Angeles, Chile. E-mail: frodriguez@formin.cl
}

\begin{abstract}
SUMMARY
Growth response of a two-year-old Pinus taeda L. (loblolly pine) plantation is evaluated for differing soil preparation and phosphorus fertilization regimes. The study was of first and second rotation sites on the Díaz de Vivar soil series, Kandihumults subgroup of the ultisols order, Corrientes Province, Argentina. A divided plot design was used in each trial, using a factorial experiment of $3 \times 2$ with three replicates. Treatment was: plowing; 50 and $80 \mathrm{~cm}$ ripping plus plowing, combined with 0 and $120 \mathrm{~g} /$ plant of triple super phosphate $(0-46-0)$. On first rotation sites, higher responses resulted from 50 and $80 \mathrm{~cm}$ ripping showing a large difference compared with plowed-only areas. At second rotation sites, no significant distinction was seen between soil preparation methods. For both site types, the application of phosphorus had a significant positive effect on growth.
\end{abstract}

Key words: Pinus taeda L., ripping, fertilization, Argentina.

\section{RESUMEN}

Se evalúa la respuesta del crecimiento de una plantación de Pinus taeda L. de dos años, bajo diferentes modalidades de roturación de suelos y fertilización con fósforo en terrenos de primera y segunda rotación de la serie Díaz de Vivar, pertenecientes al subgrupo de los Kandihumultes típicos del orden Ultisoles, del Noreste de la provincia de Corrientes, Argentina. El diseño utilizado en cada ensayo corresponde a parcelas divididas en un experimento factorial de 3 x 2 con tres repeticiones. Los tratamientos corresponden a la aplicación de rastra, subsolado a 50 y $80 \mathrm{~cm}$ de profundidad más rastra, combinados con 0 y $120 \mathrm{~g} \mathrm{pl}^{-1}$ de superfosfato triple $(0-46$ - 0$)$. Para el sector de primera rotación los mejores crecimientos se logran subsolando a 50 y $80 \mathrm{~cm}$ de profundidad, diferenciándose significativamente de las áreas preparadas sólo con rastra. En cambio, para el área de segunda rotación no se observan diferencias significativas entre los métodos de roturación de suelo. En ambos ensayos la aplicación de fósforo tuvo un efecto positivo y significativo sobre el crecimiento.

Palabras clave: Pinus taeda L., subsolado, fertilización, Argentina.

* Los resultados de este estudio forman parte de los proyectos 02 - 04 y 02 - 05, desarrollados por la Jefatura de Estudios Silvícolas de Bosques del Plata. Misiones, Argentina. 
BOSQUE 25(2): 69-76, 2004

Efectos de la roturación del suelo y fertilización con fósforo en el crecimiento...

\section{INTRODUCCION}

La región mesopotámica del Noreste de Argentina posee un gran potencial forestal y es considerada como la principal zona de bosques cultivados del país. Dentro de esta área, la provincia de Corrientes participa con un $19 \%$ de la superficie forestada del territorio argentino, que alcanza un total de 781.295 hectáreas. La mayor parte de las tierras incorporadas a la actividad forestal estaban dedicadas al uso agrícola-ganadero (1). Las especies más utilizadas son las coníferas que representan más del $90 \%$, siendo las más importantes Pinus taeda y Pinus elliottii (2).

$\mathrm{El}$ acelerado desarrollo forestal que se observa en la región, que incluye cultivos forestales intensivos, requiere una preocupación por la conservación y mejoramiento de la capacidad productiva de los sitios. Para ello, se debe tener en cuenta que el suelo y el clima son factores que determinan la productividad potencial natural del sitio, capacidad que puede ser parcialmente modificada utilizando prácticas de manipulación de suelos, como métodos de labranza diferenciados y programas de nutrición forestal adecuados para cada sitio (3, $4,5)$. Lo anterior requiere invertir en programas de investigación para determinar combinaciones efectivas de tratamientos mecánicos del suelo con el uso de fertilizantes, para lograr finalmente alcanzar las máximas ganancias en supervivencia y crecimiento de las plantaciones (6).

Entre los instrumentos más utilizados para la roturación del suelo destacan el discado y el subsolado. El discado se limita a los horizontes superficiales, mientras el subsolado reduce la densidad y aumenta la porosidad de los horizontes del subsuelo, rompiendo suelos rocosos, compactados, arcillosos, entre otros, facilitando el desarrollo radicular, la capacidad de retención de agua y la velocidad de infiltración $(7,8,9)$. Actualmente la utilización de maquinaria que combina una púa de subsolado ajustable más el discado proporciona una manipulación de suelos más completa en un solo paso, reduciendo el costo y la compactación del suelo (8).

En general, los tratamientos de labranza por sí solos no logran manifestar todo el potencial productivo de los suelos. En este contexto, las investigaciones han comprobado que la fertilización combinada con otras prácticas de cultivo compatibles con el establecimiento permiten lograr efec- tos sinérgicos que aumentan significativamente el crecimiento inicial de las plantaciones $(10,11,12)$.

La aplicación de fertilizantes en plantaciones es una práctica común en países desarrollados y está comenzando a constituir una herramienta tecnológica de importancia en países en vías de desarrollo para acelerar el crecimiento y mejorar la productividad de las plantaciones. Estas aplicaciones, generalmente, se realizan al momento del establecimiento como una fertilización de apoyo o para corregir problemas de fertilidad $(10,11,13)$.

Durante los últimos años, en el Sudeste de los Estados Unidos, las tendencias en la fertilización operacional al momento del establecimiento revelan aumentos en un 400\% desde 1995. La NCSFNC (6) reporta que durante el año 2000, sólo en plantaciones de Pinus taeda, se han fertilizado aproximadamente 567.000 hectáreas.

En Argentina no es común la fertilización durante el establecimiento de las plantaciones. Sin embargo, experiencias realizadas por Fernández et al. (1) y ensayos montados por Bosques del Plata S.A. (BdP) en 1997 y 1998, en suelos del Noreste Argentino, señalan que la labranza en conjunto con aplicaciones de fósforo tiene un efecto positivo sobre el crecimiento inicial de Pinus taeda.

Parte de la superficie de la provincia de Corrientes, donde se realizan los cultivos forestales, pertenece a la serie Díaz de Vivar, subgrupo de los Kandihumultes típicos, conocidos comúnmente como suelo de lomas rojas. Este se caracteriza, entre otros atributos, por presentar densidades superiores a los $1,3 \mathrm{~g} \mathrm{~cm}^{-3}$, alta concentración de arcilla $(9,14)$ y niveles de fósforo inferiores a 4 ppm, propiedades que evidencian la necesidad de incorporar programas de roturación de suelos y fertilización para mejorar el crecimiento y la supervivencia de las plantaciones (15).

Las experiencias para este tipo de suelo son escasas, por lo cual la Empresa Bosques del Plata S.A., que tiene un programa de forestación con Pinus taeda L. en el área, ha establecido un conjunto de ensayos para evaluar el efecto sobre el crecimiento de la preparación del suelo y determinar la viabilidad económica de los tratamientos en el largo plazo.

Específicamente el objetivo de la investigación es evaluar la respuesta del crecimiento de plantaciones de Pinus taeda L. de dos años, bajo diferentes modalidades de labranza y fertilización con fósforo en suelos de primera y segunda rotación 
BOSQUE 25(2): 69-76, 2004

Efectos de la roturación del suelo y fertilización con fósforo en el crecimiento...

de la serie Díaz de Vivar (Grupo Kandihumultes) del Noreste de la provincia de Corrientes, Argentina.

\section{MATERIAL Y METODOS}

Los ensayos se ubican al Noreste de la provincia de Corrientes, Argentina, en el predio Don Hilario, propiedad de la Empresa Forestal Bosques del Plata, a los $28^{\circ} 26^{\prime}$ de latitud sur y $55^{\circ}$ $55^{\prime}$ longitud oeste. El primer ensayo se estableció en un sector utilizado con fines agrícola-ganaderos (primera rotación) y el segundo en un área en que existió una plantación que fue afectada por fuego (segunda rotación).

Las experiencias se localizan en suelos de la serie Díaz de Vivar, la que taxonómicamente es clasificada como subgrupo de los Kandihumultes típicos del orden Ultisoles. En general, se le conoce como lomas de suelo rojo, o tierra colorada, cuya profundidad efectiva supera los $150 \mathrm{~cm}$, con pendientes que varían entre 2 a $5 \%$ y de buen drenaje (14). Se caracterizan, también, por presentar densidades superiores a los $1,3 \mathrm{~g} \mathrm{~cm}^{-3}$ (9) y por los altos contenidos de arcilla, que alcanzan un $50 \%$ en los 10 primeros centímetros, aumentando a $70 \%$ a los $80 \mathrm{~cm}$ de profundidad. El porcentaje de materia orgánica que contiene el horizonte A es de un 5\%, con un $\mathrm{pH}$ de 5,8 y valores de C.I.C. de 18,2 (14).

El clima de la zona es subtropical, muy cálido en verano, pero con heladas en invierno, siendo su temperatura promedio de $19^{\circ} \mathrm{C}$. La pluviometría media anual es de $1.631 \mathrm{~mm}$, con frecuentes excesos hídricos en otoño y primavera, mientras que en verano se presentan moderados y eventuales déficit $(14,16)$.

Las plantas utilizadas en el ensayo corresponden a Pinus taeda de origen Marion, de procedencia Florida, EE.UU., con parámetros morfológicos homogéneos (altura y diámetro), producidos en contenedores de $93 \mathrm{~cm}^{3}$. La plantación se estableció en agosto del año 2000, con un espaciamiento de $4 \mathrm{~m}$ entre líneas y 1,75 m en la línea.

Previo al establecimiento de los ensayos se realizó control de hormigas y control químico de malezas sobre toda la superficie. Además, ambos cuidados culturales se repitieron anualmente durante los meses de mayor crecimiento y competencia.
El diseño utilizado en cada ensayo corresponde a parcelas divididas en un experimento factorial de 3 x 2 con 3 repeticiones. Cada subparcela tiene un total de 108 plantas, de las cuales se evalúan las 46 centrales.

Los factores evaluados son:

- Técnicas de roturación de suelos.

- Subsolado a 50 y $80 \mathrm{~cm}$ de profundidad más dos pasadas de rastra. Para ello se utilizó un bulldozer (tipo D8) con ripper alado fijo y rastra con discos independientes retráctiles (tipo Savannah 220).

- Preparación superficial a $20 \mathrm{~cm}$ con dos pasadas de rastra, para lo cual se utilizó la rastra descrita anteriormente.

- Niveles de fertilización. Se fertilizó con superfosfato triple $(0$ - 46 - 0) en dosis de 0 y 120 $\mathrm{g} \mathrm{pl}^{-1}$. La aplicación se efectuó en dos bandas paralelas de $30 \mathrm{~cm}$ de largo, a ambos lados de la planta, en el sentido de la línea de plantación, a $15 \mathrm{~cm}$ de ésta y a $10 \mathrm{~cm}$ de profundidad. Esta se realizó en el mes de octubre del año 2000 .

El cuadro 1 detalla los seis tratamientos que resultaron de la combinación de los factores antes indicados.

Las mediciones del ensayo se realizaron al primer y segundo año de establecida la plantación. Las variables corresponden al diámetro a la altura del cuello (DAC) y altura total $(\mathrm{Ht})$. A través de las variables de control antes indicadas se determinó el crecimiento, el cual se expresa en volumen, a través del factor de productividad $\mathrm{F}_{\mathrm{p}}$.

$$
\mathrm{F}_{\mathrm{p}}=\frac{D A C^{2} \times H t}{100}
$$

Donde:

$\mathrm{F}_{\mathrm{P}} \quad=$ factor de productividad $\left(\mathrm{cm}^{3}\right)$

$\mathrm{DAC}=$ diámetro a la altura del cuello $(\mathrm{mm})$

$\mathrm{Ht}=\operatorname{altura}$ total $(\mathrm{cm})$

Los resultados obtenidos fueron analizados mediante análisis de varianza y para los tratamientos con diferencias significativas se procedió a aplicar el test de comparación de medias de Tukey. 
BOSQUE 25(2): 69-76, 2004

Efectos de la roturación del suelo y fertilización con fósforo en el crecimiento...

\section{CUADRO 1}

Descripción de los tratamientos. Treatment description.

\begin{tabular}{|l|c|c|c|}
\hline \multirow{2}{*}{ Roturación de suelo } & Fertilización $\left(\mathrm{g} \mathrm{pl}^{-1}\right)$ & Tratamiento & Rótulo \\
\hline \multirow{2}{*}{$\begin{array}{l}\text { Subsolado a } 50 \mathrm{~cm} \text { de profundidad } \\
+2 \text { pasadas de rastra }\end{array}$} & 0 & 1 & $50-\mathrm{S}$ \\
\cline { 2 - 4 } & 120 & 2 & $50-\mathrm{C}$ \\
\hline $\begin{array}{l}\text { Subsolado a } 80 \mathrm{~cm} \text { de profundidad } \\
+2 \text { pasadas de rastra }\end{array}$ & 0 & 3 & $80-\mathrm{S}$ \\
\cline { 2 - 4 } 2 pasadas de rastra & 120 & 4 & $80-\mathrm{C}$ \\
\cline { 2 - 4 } & 0 & 5 & $20-\mathrm{S}$ \\
\hline
\end{tabular}

\section{RESULTADOS Y DISCUSION}

Respuesta inicial a la preparación de suelo en sitios de primera rotación: La evaluación del crecimiento de las variables DAC, Ht y $\mathrm{F}_{\mathrm{p}}$ señalan que los tratamientos con subsolado a $50 \mathrm{~cm}$ y a $80 \mathrm{~cm}$ de profundidad son mayores y se diferencian significativamente del tratamiento de preparación con rastra a $20 \mathrm{~cm}$ (cuadro 2). Un comportamiento similar entre el subsolado y aplicación de rastra reporta Fernández et al. (5) en plantaciones de $P$. taeda localizadas en suelos pertenecientes al gran grupo de los Kandiudalfes. También, en plantaciones de Pinus radiata D. Don, Flinn y Aeberli (17) y Mead (18) reportan que el subsolado a profundidades de $0,6 \mathrm{~m}$ aumenta notablemente el crecimiento en volumen a edades tempranas, diferenciándose significativamente de la preparación sólo con rastra.

Al subsolar a $50 \mathrm{~cm}$ de profundidad las ganancias en crecimiento para las variables DAC y Ht son de $12,6 \%(8,5 \mathrm{~mm})$ y $12,2 \%(25,6 \mathrm{~cm})$, respectivamente, si se compara con la roturación del terreno sólo con rastra. Fernández et al. (5) realizan una comparación similar en una plantación de 37 meses y obtienen ganancias de un $7 \%$ para la variable DAC y $8 \%$ para la Ht. En general, se puede establecer para el ensayo que las ganancias generadas durante los dos primeros años, producto de las roturaciones en profundidad, tienden a disminuir en el tiempo.

\section{CUADRO 2}

Efectos de la roturación del suelo sobre el crecimiento de Pinus taeda a los dos años, en sitios de primera rotación.

Effects of soil ripping in first rotation sites with two-year-old Pinus taeda plantations.

\begin{tabular}{|l|c|c|c|c|c|c|}
\hline \multirow{2}{*}{$\begin{array}{l}\text { Niveles del factor } \\
\text { Roturación de suelo }\end{array}$} & \multicolumn{2}{|c|}{ Media DAC $(\mathrm{mm})$} & \multicolumn{2}{c|}{ Media Ht $(\mathrm{cm})$} & \multicolumn{2}{c|}{ Media $\mathrm{F}_{\mathrm{p}}\left(\mathrm{cm}^{3}\right)$} \\
\cline { 2 - 7 } & Año 1 & Año 2 & Año 1 & Año 2 & Año 1 & Año 2 \\
\hline Subsolado $50 \mathrm{~cm}$ & $27,0 \mathrm{a}$ & $76,5 \mathrm{a}$ & $86,5 \mathrm{a}$ & $236,5 \mathrm{a}$ & $730,1 \mathrm{a}$ & $14.526,9 \mathrm{a}$ \\
Subsolado $80 \mathrm{~cm}$ & $26,6 \mathrm{a}$ & $76,2 \mathrm{a}$ & $82,8 \mathrm{a}$ & $231,7 \mathrm{~b}$ & $718,3 \mathrm{a}$ & $14.199,8 \mathrm{a}$ \\
Rastra $20 \mathrm{~cm}$ & $20,8 \mathrm{~b}$ & $68,0 \mathrm{~b}$ & $72,2 \mathrm{~b}$ & $210,8 \mathrm{c}$ & $399,0 \mathrm{~b}$ & $10.537,8 \mathrm{~b}$ \\
\hline
\end{tabular}

Nota: letras distintas indican diferencias estadísticas según el test de Tukey (5\%). 
La ausencia de diferencias significativas entre las distintas profundidades de subsolado manifestadas en este ensayo, sugiere excluir el subsolado de $80 \mathrm{~cm}$ de las labores de establecimiento, más aún cuando éste exhibe valores inferiores a los logrados con $50 \mathrm{~cm}$ (cuadro 2, figura 1).

En relación a la fertilización con fósforo, se determinó que los tratamientos en que se adicionó $120 \mathrm{~g} \mathrm{pl}^{-1}$ de SFT presentan mayores crecimientos en $\mathrm{DAC}$, Ht y $\mathrm{F}_{\mathrm{p}}$, diferenciándose significativamente de las áreas no tratadas (cuadro 3). Fernández et al. (19) y BdP (15) obtuvieron respuestas similares en plantaciones de $P$. taeda creciendo en suelos de primera rotación del grupo Kandiudalfes. Resultados equivalentes

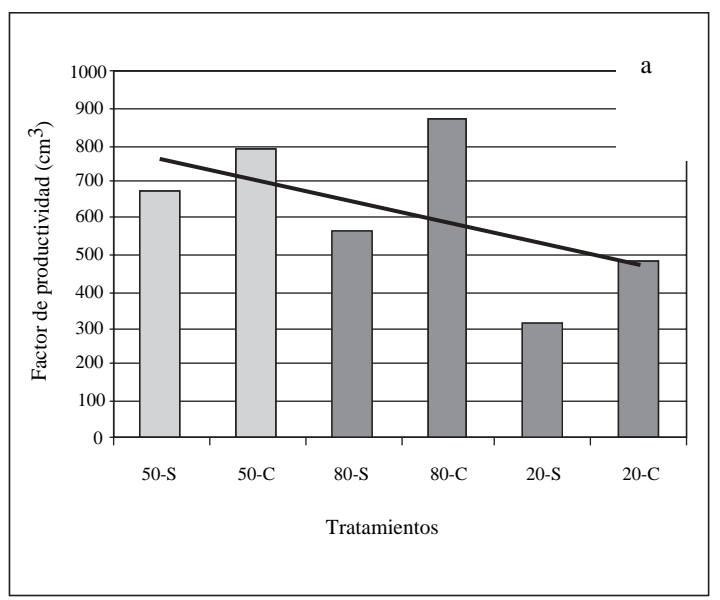

para la misma especie, creciendo en otros sitios, han sido obtenidos por FORMIN (20), Allen y Albaugh (21) y NCSFNC (6).

A dos años de establecido el ensayo las ganancias en crecimiento de las variables DAC y $\mathrm{Ht}$, producto de la fertilización con fósforo, son de $5,9 \%(4,2 \mathrm{~mm})$ y $5,5 \%(12,2 \mathrm{~cm})$ respectivamente. Los incrementos anuales por fertilización tienden a disminuir, al igual que para la roturación del suelo.

La supervivencia no mostró respuesta a los factores evaluados (roturación de suelo y fertilización). En general, todos los tratamientos presentan buenos índices de supervivencia (> 89,1\%), permaneciendo constantes para el primer y segundo año.

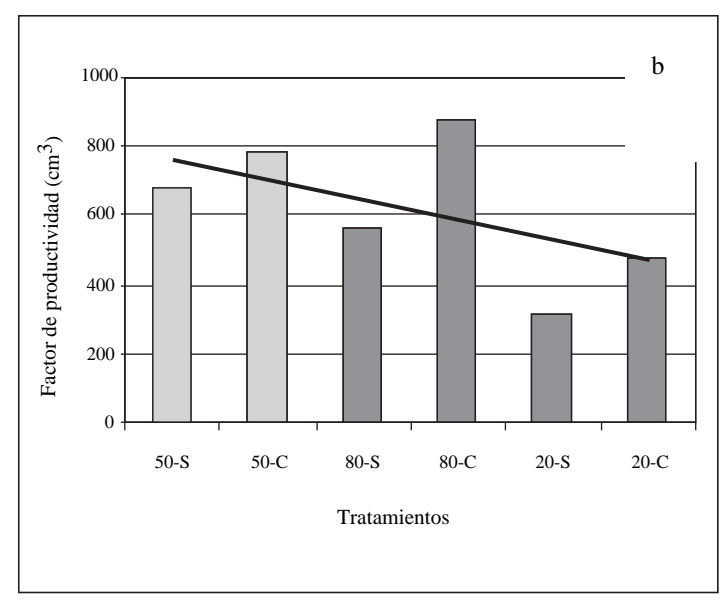

Figura 1. Factor de productividad $\left(\mathrm{cm}^{3}\right)$, según tratamiento, para sitios de primera rotación: a) Evaluación al primer año; b) Evaluación al segundo año.

Productivity factor $\left(\mathrm{cm}^{3}\right)$ for first rotation sites, by treatment: a) first-year evaluation, b) second-year evaluation.

\section{CUADRO 3}

Efectos de la fertilización (SFT) sobre el crecimiento de Pinus taeda a los dos años, en sitios de primera rotación.

Fertilization effects on growth of two-year-old Pinus taeda seedlings in first rotation sites.

\begin{tabular}{|l|c|c|c|c|c|c|}
\hline \multirow{2}{*}{$\begin{array}{c}\text { Niveles del factor } \\
\text { Fertilización }\end{array}$} & \multicolumn{2}{|c|}{ Media DAC (mm) } & \multicolumn{2}{c|}{ Media Ht (cm) } & \multicolumn{2}{c|}{ Media $\mathrm{F}_{\mathrm{p}}\left(\mathrm{cm}^{3}\right)$} \\
\cline { 2 - 6 } & Año 1 & Año 2 & Año 1 & Año 2 & Año 1 & Año 2 \\
\hline $120 \mathrm{~g} \mathrm{pl}^{-1}$ & $26,6 \mathrm{a}$ & $75,7 \mathrm{a}$ & $84,3 \mathrm{a}$ & $232,4 \mathrm{a}$ & $712,7 \mathrm{a}$ & $14.087,7 \mathrm{a}$ \\
$0 \mathrm{~g} \mathrm{pl}^{-1}$ & $23,1 \mathrm{~b}$ & $71,5 \mathrm{~b}$ & $76,8 \mathrm{~b}$ & $220,2 \mathrm{~b}$ & $518,9 \mathrm{~b}$ & $12.088,7 \mathrm{~b}$ \\
\hline
\end{tabular}

Nota: letras distintas indican diferencias estadísticas según el test de Tukey (5\%). 
BOSQUE 25(2): 69-76, 2004

Efectos de la roturación del suelo y fertilización con fósforo en el crecimiento...

Dado que la interacción de los factores no es significativa, los efectos sólo se adicionan. En este contexto, la respuesta aditiva de los factores se encuentra expresada claramente en la figura 1, en la que se observa que los tratamientos con roturación de suelo presentan mayores crecimientos cuando se le suministra fósforo. Al respecto, Ballard (22) señala que cuando se mejoran las condiciones físicas del suelo la sensibilidad a la aplicación del fósforo aumenta notablemente, sobre todo en suelos arcillosos.

Respuesta inicial a la preparación de suelo en sitios de segunda rotación: A diferencia de lo ocurrido en sitios de primera rotación, el efecto sobre el crecimiento de los métodos de roturación no es significativo (cuadro 4). Un comportamiento similar reportó Lupi et al. (23) en sitios de segunda rotación con plantaciones de 9 meses de Eucalyptus grandis Hill ex Maiden, creciendo en suelos del gran grupo Kandiudalfes.

La ausencia de diferencias estadísticas entre las distintas modalidades de roturación del suelo podría estar ligada al aumento de la porosidad, producto de la muerte y descomposición de los sistemas radiculares del rodal original del área, que mejoran la infiltración, el contenido de materia orgánica, la aireación, entre otros (24).

Al no existir, a la fecha, diferencias entre los tratamientos de roturación de suelo, la preparación con rastra se presenta como una técnica adecuada para zonas de segunda rotación (cuadro 4, figura 2).

\section{CUADRO 4}

Efectos de la roturación del suelo sobre el crecimiento de Pinus taeda a los dos años, en sitios de segunda rotación.

Soil ripping effects on growth of two-year-old seedlings in second rotation sites.

\begin{tabular}{|l|c|c|c|c|c|c|}
\hline \multirow{2}{*}{$\begin{array}{l}\text { Niveles del factor } \\
\text { Roturación de suelo }\end{array}$} & \multicolumn{2}{|c|}{ Media DAC $(\mathrm{mm})$} & \multicolumn{2}{c|}{ Media Ht $(\mathrm{cm})$} & \multicolumn{2}{c|}{ Media $\mathrm{F}_{\mathrm{p}}\left(\mathrm{cm}^{3}\right)$} \\
\cline { 2 - 7 } & Año 1 & Año 2 & Año 1 & Año 2 & Año 1 & Año 2 \\
\hline Subsolado $50 \mathrm{~cm}$ & $30,2 \mathrm{a}$ & $77,8 \mathrm{a}$ & $90,8 \mathrm{a}$ & $235,4 \mathrm{a}$ & $899,2 \mathrm{a}$ & $14.817,9 \mathrm{a}$ \\
Subsolado $80 \mathrm{~cm}$ & $27,8 \mathrm{a}$ & $74,6 \mathrm{a}$ & $87,1 \mathrm{a}$ & $233,1 \mathrm{a}$ & $785,9 \mathrm{a}$ & $13.732,9 \mathrm{a}$ \\
Rastra $20 \mathrm{~cm}$ & $27,9 \mathrm{a}$ & $75,3 \mathrm{a}$ & $86,5 \mathrm{a}$ & $238,4 \mathrm{a}$ & $817,3 \mathrm{a}$ & $14.536,6 \mathrm{a}$ \\
\hline
\end{tabular}

Nota: letras distintas indican diferencias estadísticas según el test de Tukey (5\%).
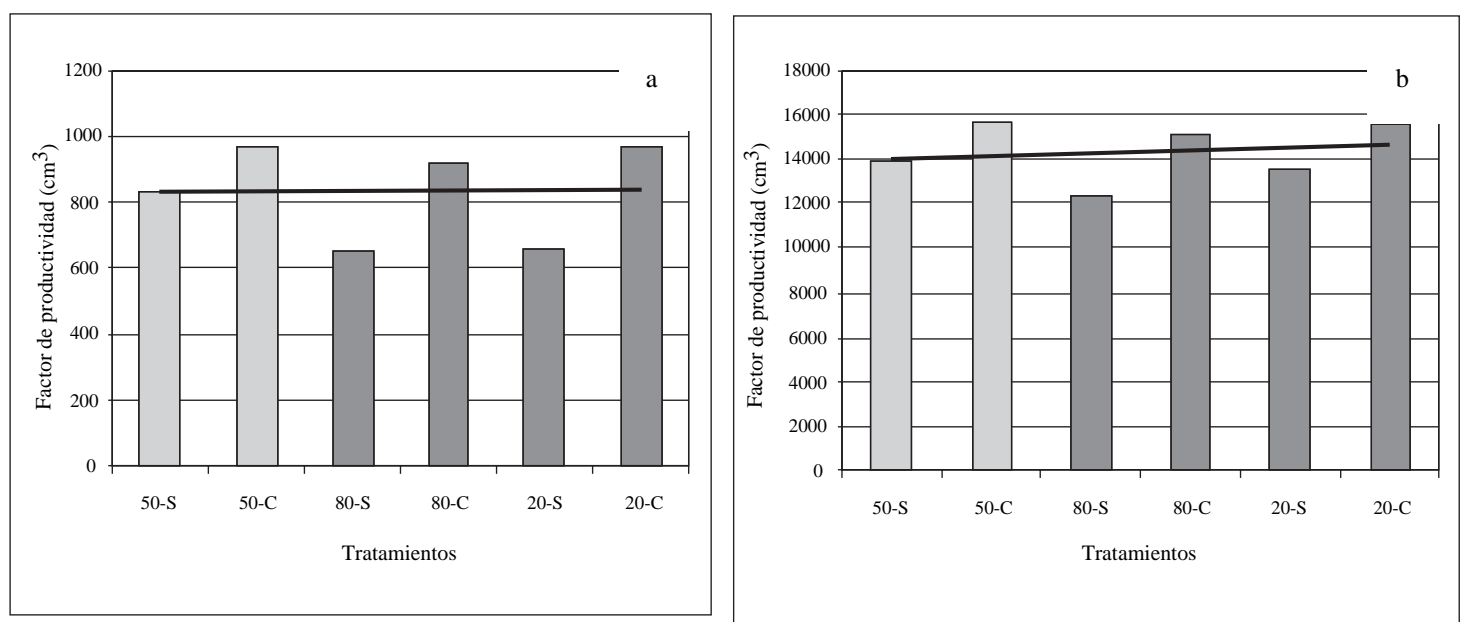

Figura 2. Factor de productividad $\left(\mathrm{cm}^{3}\right)$, según tratamiento, para sitios de segunda rotación: a) Evaluación al primer año; b) Evaluación al segundo año.

Productivity factor $\left(\mathrm{cm}^{3}\right)$ for second rotation sites, by treatment: a) first-year evaluation, b) second-year evaluation. 
BOSQUE 25(2): 69-76, 2004

Efectos de la roturación del suelo y fertilización con fósforo en el crecimiento..

\section{CUADRO 5}

Efectos de la fertilización (SFT) sobre el crecimiento de Pinus taeda a los dos años, en sitios de segunda rotación.

Fertilization effects on growth of two-year-old Pinus taeda seedlings on second rotation.

\begin{tabular}{|c|c|c|c|c|c|c|}
\hline \multirow{2}{*}{$\begin{array}{c}\text { Niveles del factor } \\
\text { Fertilización }\end{array}$} & \multicolumn{2}{|c|}{ Media DAC (mm) } & \multicolumn{2}{c|}{ Media Ht $(\mathrm{cm})$} & \multicolumn{2}{c|}{ Media $\mathrm{F}_{\mathrm{p}}\left(\mathrm{cm}^{3}\right)$} \\
\cline { 2 - 6 } & Año 1 & Año 2 & Año 1 & Año 2 & Año 1 & Año 2 \\
\hline $120 \mathrm{~g} \mathrm{pl}^{-1}$ & $30,0 \mathrm{a}$ & $77,5 \mathrm{a}$ & $91,0 \mathrm{a}$ & $243,1 \mathrm{a}$ & $954,7 \mathrm{a}$ & $15.451,7 \mathrm{a}$ \\
$0 \mathrm{~g} \mathrm{pl}^{-1}$ & $27,2 \mathrm{~b}$ & $74,3 \mathrm{~b}$ & $85,3 \mathrm{~b}$ & $228,1 \mathrm{~b}$ & $713,6 \mathrm{~b}$ & $13.273,3 \mathrm{~b}$ \\
\hline
\end{tabular}

Nota: letras distintas indican diferencias estadísticas según el test de Tukey (5\%).

En relación a los tratamientos con aplicaciones de fósforo, éstos presentan mayores crecimientos y se diferencian significativamente de los no fertilizados (cuadro 5). Por efecto de fertilización se obtiene, al segundo año de establecida la plantación, un aumento en el factor de productividad $\left(\mathrm{F}_{\mathrm{p}}\right)$ de $16,4 \%\left(2.178,4 \mathrm{~cm}^{3}\right)$. Al igual que para los sitios de primera rotación los incrementos anuales tienden a disminuir.

El índice de supervivencia para estos sitios es en general alto $(>86,3 \%)$ y no es afectado por las técnicas de roturación de suelos ni por la fertilización con fósforo, que influyen solamente en el crecimiento.

\section{CONCLUSIONES}

El análisis de los resultados, a dos años de efectuada la plantación, indica que para sitios de primera rotación los mejores crecimientos se logran fertilizando con $120 \mathrm{~g} \mathrm{pl}^{-1}$ de superfosfato triple y roturando el suelo a $50 \mathrm{~cm}$ de profundidad más dos pasadas de rastra.

En el sitio de segunda rotación sólo mostró significancia el factor fertilización, específicamente el aporte de $120 \mathrm{~g} \mathrm{pl}^{-1}$ de superfosfato triple.

Debido a la disminución del efecto de los tratamientos que es apreciado en el ensayo, se debe continuar la evaluación de estos, de manera de poder determinar la viabilidad económica de realizar las prácticas descritas.

\section{BIBLIOGRAFIA}

(1) FERNANDEZ, R., F. RODRIGUEZ, A. LUPI, A. HERNANDEZ, H. REIS. Efectos de diferentes prácticas de preparación del terreno y fertilización sobre el crecimiento inicial del Pinus spp en el NE argentino. Bosque (Chile). 1999, vol. 20, $\mathrm{N}^{\mathrm{o}}$ 1, p. 47-55.

(2) DALLA-TEA, F., M. MARCO. El cultivo del Eucalipto en la Mesopotamia Argentina. En: Actas Simposio, Los Eucaliptus en el desarrollo forestal de Chile. Pucón, Chile. 1993 , p. $475-484$.

(3) BINKLEY, D. Nutrición Forestal: Prácticas de manejo. $1^{a}$ ed. Editorial Limusa. 1993. México. 518 p.

(4) TRIEBWASSER, M., S. ALTSULER. Fertilization practices and application procedures at weyerhaeuser. In: LANDIS, T. D.; GREGG, B., tech. coords. National. Proceedings, Forest and Conservation Nursery Associations. Gen. Tech. Rep. PNW-GTR 365. Portland, OR: US. Department of Agriculture, Forest Service, Pacific Northwest Research Station. 1995, p. 84-88.

(5) FERNANDEZ, R., A. LUPI, F. RODRIGUEZ, M. GELID, R. PEZZUTTI, N. PAHR, A. HERNANDEZ, H. DELGADINO, P. CORTEZ. Respuesta de Pinus taeda L. a diferentes modalidades de roturación en áreas de suelos rojos. Trabajo presentado en las Jornadas Silvoargentinas: Avances en el establecimiento de plantaciones de coníferas Subtropicales en el MERCOSUR. Virasoro, Argentina. 2000. 7 p.

(6) NORTH CAROLINA STATE FOREST NUTRITION COOPERATIVE (NCSFNC). North Carolina State University, Department of Forestry. Thirtieth annual report. June 2001. 14 p.

(7) LARRAIN, O. Establecimiento de plantaciones de Eucaliptus en forestal Angol Limitada. En: Actas de simposio, Los Eucaliptus en el desarrollo forestal de Chile. Pucón, Chile. 1993, p. 157-165.

(8) NORTH CAROLINA STATE FOREST NUTRITION COOPERATIVE (NCSFNC). Early Loblolly Pine growth response to surface and subsurface tillage. College of forest resources. North Carolina State University. Report $N^{\circ} 14$ 2000. 42 p.

(9) RODRIGUEZ, F. El paquete tecnológico aplicado por Bosques del Plata S.A. XVI Jornadas Forestales de Entre Ríos. Entre Ríos, Argentina. 2001. 28 p. 
BOSQUE 25(2): 69-76, 2004

Efectos de la roturación del suelo y fertilización con fósforo en el crecimiento...

(10) TORO, J. Avances en fertilización en Pino y Eucaliptus en Chile. Simposio IUFRO: Manejo nutritivo de plantaciones forestales. Valdivia, Chile. 1995, p. 293-299.

(11) QUIJADA, M. Fertilización en plantaciones de Pinus radiata D. Don en Chile. Tesis Ingeniero Ejecución Forestal. Corporación Universidad de Concepción. Los Angeles, Chile. 1997. 122 p.

(12) ALVAREZ, J., J. RODRIGUEZ, D. SUAREZ. Mejoramiento de la productividad de plantaciones de Pinus radiata $\mathrm{D}$. Don, a través de un método racional de fertilización. Bosque (Chile). 1999, vol. 20, № 1, p. 23-36.

(13) ALVAREZ, J. Avances en fertilización de Plantaciones en Forestal Mininco S.A. VI Jornadas de Establecimiento de Plantaciones: Aporte de los silvicultores a la competitividad de Forestal Mininco a través de la excelencia en el establecimiento de plantaciones. Temuco, Chile. 2000. 38 p.

(14) ESCOBAR, E., H. LIGIER, R. MELGAR, H. MATTEIO, O. VALLEJOS. Suelos. Mapa de suelos para la provincia de Corrientes. INTA, Subsecretaría de Recursos Naturales y Medio Ambiente. Corrientes, Argentina. 1996. 432 p.

(15) BOSQUES DEL PLATA (BDP). Informe: Resultados de Ensayos silvícolas realizados en Bosques del Plata. Superintendencia de Silvicultura, Departamento de Soporte Técnico. Posadas, Argentina. 2001. 300 p.

(16) MIRO, P. Informe climatológico: Estaciones VirasoroSanto Tomé. Bosques del Plata, División silvícola. Apóstoles (Argentina). 2001. 29 p.

(17) FLINN, D., B. AEBERLI. Establishment techniques for radiata pine on poorly drained soils deficient in phosphorus. Australian Forestry. 1982, vol. 45, № 3, p. 164-173.
(18) MEAD, D. Response of young Pinus radiata to cultivation and fertiliser Near Motueka, New Zealand. New Zealand Journal of Forestry Science. 1990, vol. 20, № 3, p. 268-278.

(19) FERNANDEZ, R., F. RODRIGUEZ, A. LUPI, E. LOPEZ, R. PEZZUTTI, E. CRECHI, N. PAHR, M. NATIUCK, P. CORTEZ. Respuesta de Pinus taeda L. a la fertilización inicial con N, P y K. Trabajo presentado en las Jornadas Silvoargentinas: Avances en el establecimiento de plantaciones de coníferas Subtropicales en el MERCOSUR. Virasoro, Argentina. 2000. 9 p.

(20) FORESTAL MININCO S.A. (FORMIN). Bio-ecología, mejoramiento genético y principios de silvicultura y manejo de la especie Pinus taeda L. (Loblolly Pine). Los Angeles. Chile. 1995. 36 p.

(21) ALLEN, H., T. ALBAUGH. Ecophysiological for plantation production: A loblolly pine case study. Bosque (Chile). 1999, vol. 20, $\mathrm{N}^{\circ} 1$, p. 3-8.

(22) BALLARD, R. Effect of first rotation phosphorus applications on fertiliser requirements of second rotation radiata pine. New Zealand Journal of Forestry Science. 1978, vol. 8, $\mathrm{N}^{\mathrm{o}} 1$, p. $135-145$

(23) LUPI, A., R. FERNANDEZ, H. REIS, J. BERNIO, J. ELIZAUL, H. REBORATTI. Evaluación inicial de técnicas de establecimiento postala rasa sobre el crecimiento inicial del Eucalyptus grandis hill. ex maiden en el Noreste Argentino. Documento resumen en: Actas de Congreso Mundial IUFRO (Malasia). 2000.

(24) DONOSO, C. Ecología Forestal: El Bosque y su medio ambiente. $5^{\text {a }}$ ed. Santiago, Chile. Editorial Universitaria. 1997. $369 \mathrm{p}$. 